ISSN 0103-5150

Fisioter. Mov., Curitiba, v. 26, n. 1, p. 203-217, jan./mar. 2013

Licenciado sob uma Licença Creative Commons

\title{
Instrumentos de avaliação da postura dinâmica: aplicabilidade ao ambiente escolar
}

\author{
Tools for evaluation the dynamic posture: \\ applicability to the school environment
}

\section{Matias Noll[ ${ }^{[a]}$, Cláudia Tarragô Candotti ${ }^{[b]}$, Adriane Vieira ${ }^{[c]}$}

[a] Mestre em Ciências do Movimento Humano pela Universidade Federal do Rio Grande do Sul (UFRGS), Porto Alegre, RS Brasil, e-mail: matiasnoll@yahoo.com.br

[b] Doutora em Ciências do Movimento Humano pela Universidade Federal do Rio Grande do Sul (UFRGS), professora do curso de Fisioterapia e Educação Física, e do Programa de Pós-Graduação (Mestrado e Doutorado) em Ciências do Movimento Humano da Universidade Federal do Rio Grande do Sul (UFRGS), Porto Alegre, RS - Brasil, e-mail: claudia.candotti@ufrgs.br

[c] Doutora em Ciências do Movimento Humano pela Universidade Federal do Rio Grande do Sul (UFRGS), professora do curso de Fisioterapia e de Educação Física da Universidade Federal do Rio Grande do Sul (UFRGS), Porto Alegre, RS Brasil, e-mail: adriane.vieira@gmail.com

\section{Resumo}

Introdução:Para que a avaliação da postura dinâmica seja efetivada é necessário, primeiramente, conhecer os diversos instrumentos, disponíveis e validados na literatura, apropriados para esse fim. Objetivo: O objetivo deste artigo de revisão sistemática foi descrever, sintetizar e analisar criticamente os instrumentos encontrados na literatura que objetivem avaliar a postura dinâmica, tanto em adultos quanto em escolares, e refletir sobre a possibilidade de utilização desses métodos no ambiente escolar. Materiais e métodos: Foi realizada uma busca sistemática de artigos em bases de dados (Scopus, ScienceDirect, PubMed, SciELO) publicados a partir da década de 1980 e no Banco de Teses e Dissertações da Capes. As palavras-chave utilizadas na busca pelos artigos foram back, spine, back injuries, school, back school, postural hygiene program, education, child, student, posture, em combinação com as palavras-chave evaluation, assessment, measurement, e os respectivos termos em português. Os instrumentos propostos deveriam preencher os seguintes critérios: (a) avaliar a postura corporal durante a realização de atividades da vida diária (AVDs); (b) utilizar 
critérios pré-definidos de avaliação da postura dinâmica; e (c) avaliar a postura a partir de observação, direta ou a partir de filmagem. Resultados: Foram identificados oito artigos originais que apresentam instrumentos de avaliação da postura dinâmica, avaliando a execução de AVDs a partir de critérios biomecânicos pré-definidos por escalas numéricas; destes, apenas quatro instrumentos foram elaborados com o propósito de avaliar a execução de AVDs de escolares. Considerações finais: Em geral, os instrumentos apresentam algumas limitações metodológicas, embora sejam de fácil aplicabilidade.

Palavras-chave: Avaliação. Postura. Movimento. Adolescente.

\section{Abstract}

Introduction: To make the evaluation of dynamic posture, it is necessary, first, get to know the various tools, available and validated in the literature, appropriate for this purpose. Objective: To describe, summarize and critically analyze the instruments found in the literature that aim to evaluate the dynamic posture, both in adults and schoolchildren, and reflect on the possible use of these methods in the school environment. Materials and methods: It's performed a systematic search of databases of articles (Scopus, ScienceDirect, PubMed, SciELO) published since the 1980 and in the Banco de Teses e Dissertações da CAPES. The keywords used in search for articles were: "back", "spine", "back injuries", "school", "back school", "postural hygiene program", "education", "child", "student, posture", in combination with the keywords "evaluation", "assessment", "measurement", and the corresponding terms in Portuguese. The instruments proposed should meet the following criteria: (a) to assess the body posture during activities of daily living (ADLS) (b) to use pre-defined criteria for evaluating the dynamic posture, and (c) to assess the posture from observation, either directly or from shooting. Results: It's identified eight original articles that present tools for evaluating the dynamic posture, assessing the ADLs through biomechanical criteria predefined by means of numerical scales, but only four instruments have been drafted in order to assess ADLs schoolchildren. Conclusions: In general, the instruments are some methodological limitations, although they are easy to apply.

Keywords: Evaluation. Posture. Motion. Adolescent.

\section{Introdução}

Os problemas posturais e a ocorrência de dor estão disseminados entre a população em geral $(1,2,3)$, manifestando-se também em grandes proporções na infância e na adolescência $(3,4,5)$. Já foi bem documentado na literatura que a ocorrência de dor musculoesquelética em duas ou mais áreas anatômicas é muito frequente em jovens escolares $(6,7)$, assim como a prevalência de alterações posturais da coluna vertebral, tanto laterais quanto anteroposteriores, podendo acometer mais de $60 \%$ dos avaliados nesta população (8).

Esses problemas em jovens, tanto a dor nas costas quanto as alterações posturais, podem, segundo Siivola et al. (9), ter causa multifatorial. São identificados como fatores de risco para o surgimento da lombalgia e dos problemas posturais em jovens: utilização de mochilas pesadas e transporte de modo assimétrico, permanência por longos períodos de tempo em postura inadequada durante a posição sentada (9), utilização de mobílias inadequadas, permanência por muito tempo em frente à televisão (10), realização de diferentes Atividades de Vida Diária (AVDs) com postura inadequada (11), menos de sete horas de sono por dia, tabagismo, sobrepeso (12) e apresentação de algum fator psicossocial, como por exemplo, depressão e ansiedade (4), entre outros,.

Visto que a postura corporal executada durante as AVDs está presente entre os fatores de risco associados à lombalgia e à ocorrência de problemas posturais $(13,14)$, especula-se que ela tenha importantes implicações para a saúde e o bem-estar do ser humano. Isso porque ela determina a quantidade e a distribuição do esforço sobre os vários ossos, músculos, tendões, ligamentos e discos intervertebrais, podendo potencializar ou amenizar os malefícios e sobrecargas resultantes na coluna vertebral (2). Assim, sabendo-se que um grande percentual das alterações posturais e das dores lombares é causado pela falta de hábitos posturais adequados durante as 
AVDs $(1,14)$, é relevante investigá-la já na infância e juventude, pois a postura adequada e a correção precoce de alterações posturais nessa fase possibilitam padrões posturais adequados na vida adulta (13, 15-18). A partir desse pressuposto, assume-se que a idade escolar compreende a fase ideal para recuperar disfunções da coluna vertebral de maneira eficaz, pois após esse período, o prognóstico torna-se mais difícil e o tratamento mais prolongado (14).

$\mathrm{Na}$ literatura, encontram-se alguns estudos com a população de escolares que investigam a dor lombar, problemas posturais e fatores associados (6-10). Mais especificamente, tais estudos objetivam avaliar o local, a frequência e a intensidade da dor e os possíveis fatores de risco (gênero, tempo diário de uso de computador, tempo ininterrupto e/ou diário na posição sentada, consumo de cigarros, meio e modo de transporte do material escolar, nível de atividade física, índice de massa corporal, entre outros). Entretanto, a maioria desses estudos não tem se preocupado em avaliar a postura corporal durante a execução de AVDs $(19,20,21)$.

Nos poucos estudos encontrados em que é avaliada a postura corporal durante as AVDs de escolares, sejam estudos descritivos transversais $(8,21,22)$ ou longitudinais (17), o método largamente utilizado para avaliar a postura dinâmica é o questionário. Do mesmo modo, em publicações científicas sobre Escolas Posturais, denominadas também de Escola de Postura, Escola de Coluna ou Back School, que objetivam a diminuição da dor pela incorporação de formas mais adequadas de executar AVDs, predomina a utilização de questionários para avaliação da qualidade de vida $(23,24)$, da dor $(25)$, da funcionalidade $(26$, $27)$ e da postura corporal as AVDs $(28,29)$. Isso ocorre em detrimento de instrumentos como circuitos de AVDs, os quais avaliam a postura dinâmica por meio da observação visual e/ou filmagem $(15,16,30)$.

Referente a esse tema, Candotti et al. (20), Spence et al. (14) e Andreotti e Okuma (31) relatam que há necessidade de utilizar conjuntamente os questionários e os testes dinâmicos padronizados para avaliação da postura dinâmica, elevando a qualidade da avaliação. Não obstante, Thomas et al. (32) destacam que, além da padronização e adequação ao contexto do avaliado, esse tipo de instrumento deve ter sua validade (concorrente e/ou de conteúdo e de constructo) e reprodutibilidade intra-avaliador e interavaliador e repetibilidade confirmadas ${ }^{1}$. Nesse sentido, qualquer instrumento que se proponha a avaliar a postura dinâmica deveria contemplar minimamente algumas dessas características para que o avaliador tenha segurança de que os resultados representem as posturas adotadas pelos avaliados.

A literatura apresenta ainda outras técnicas mais sofisticadas que possibilitam avaliar a execução de AVDs, como a cinemetria (33), eletromiografia (34), dinamometria $(35,36,37)$, e ressonância magnética (21). Entretanto, os professores e profissionais especializados que desenvolvem avaliações no ambiente escolar não detêm a tecnologia necessária para essas avaliações, pois são de alto custo, de difícil adaptação ao ambiente escolar e requererem técnicos especializados para sua operacionalização (38). Tal fato reforça a importância da avaliação da postura dinâmica no contexto escolar por observação visual e/ou filmagem, visto que possibilitam avaliar os escolares fora do ambiente laboratorial.

Desse modo, para que a investigação da postura dinâmica seja efetivada, é necessário, primeiramente, conhecer os diversos instrumentos disponíveis e apropriados na literatura para esse fim. Além disso, independente do contexto em que é realizada a avaliação da postura dinâmica (as próprias escolas ou escolas posturais) e do tipo de protocolo dessa avaliação, é fundamental que a metodologia escolhida seja confiável para fornecer maior qualidade aos resultados $(16,20)$. Deve-se, ainda, avaliar a execução de AVDs mais relevantes, ou seja, aquelas realizadas mais frequentemente no dia a dia dos jovens, levando em consideração aspectos como a imposição de sobrecargas (mochilas, pastas etc.) e o tempo de exposição às mesmas (14). Dessa forma, justifica-se este artigo de revisão, com o objetivo de descrever,

\footnotetext{
1 Validade refere-se ao grau de veracidade das medições de uma determinada grandeza. Reprodutibilidade interavaliador refere-se ao grau de reprodutibilidade das medições de uma mesma grandeza quando se repetem as medidas variando o observador, mantendo as demais condições constantes, sendo as medições realizadas em instantes sucessivos. Reprodutibilidade intra-avaliador refere-se ao grau de reprodutibilidade das medições de uma mesma grandeza quando se repetem as medidas variando o tempo entre as coletas, mantendo as demais condições constantes (mesmo observador), sendo que as coletas devem ser realizadas com intervalo mínimo de um dia. Repetibilidade refere-se ao grau de concordância entre o resultado de medições sucessivas de uma grandeza, efetuada com a totalidade das seguintes condições: igual método de medição, o mesmo observador, o mesmo instrumento de avaliação, o mesmo local, as mesmas condições de utilização e repetição em instantes sucessivos $(39,40)$.
} 
sintetizar e analisar criticamente os instrumentos encontrados na literatura que visam avaliar a postura dinâmica, tanto em adultos quanto em escolares, e refletir sobre a possibilidade de utilização desses métodos no ambiente escolar.

\section{Metodologia}

Para cumprir com o propósito do estudo, foi realizada uma busca sistemática de artigos científicos publicados a partir da década de 1980 em várias bases de dados (Scopus, ScienceDirect, PubMed, SciELO), além de dissertações e teses, no Banco de Teses e Dissertações da Capes. As palavras-chave utilizadas na busca pelos artigos foram back, spine, back injuries, school, back school, postural hygiene program, education, child, student, posture, em combinação com as palavras-chave evaluation, assessment e measurement, e os respectivos termos em português.

Os instrumentos propostos nas publicações encontradas, para integrar a presente revisão de literatura, deveriam preencher os seguintes critérios: (a) avaliar a postura durante as AVDs; (b) utilizar critérios pré-definidos de avaliação da postura dinâmica; e (c) avaliar a postura a partir de observação, seja direta ou a partir de filmagem. Foram excluídos da presente revisão os estudos que não atenderam a três critérios estipulados. Foram identificados, a partir desse procedimento, oito artigos originais que estavam adequados aos critérios estabelecidos e que são analisados nesta revisão.

\section{Avaliação da postura corporal dinâmica}

Instrumentos de avaliação

A busca realizada nas bases de dados resultou na seleção de oito artigos originais, os quais cumpriram com todos os critérios de inclusão. No entanto, não foram encontradas dissertações e teses que pudessem ser incluídas no presente artigo de revisão. Os instrumentos propostos nas publicações analisadas foram avaliados em relação à validação (aspectos amostrais e metodológicos) e a sua aplicabilidade no ambiente escolar. Desenvolveu-se também uma breve discussão e reflexão sobre cada um deles e sobre suas principais características. No Quadro 1 estão apresentados todos os artigos que cumpriram com os critérios de seleção, ressaltando-se os respectivos aspectos amostrais e metodológicos, tais como: as posturas analisadas e o procedimento de validação e seus resultados estatísticos, além dos artigos nos quais o instrumento foi utilizado. A seguir listar-se-ão os artigos por ordem cronológica, assim como uma breve discussão e reflexão sobre cada um deles e suas principais características.

0 primeiro artigo encontrado na literatura que analisa as AVDs por meio de observação foi o trabalho de Spence et al. (14), realizado com escolares. Esse estudo objetivou avaliar os efeitos de um método de ensino da técnica adequada de levantamento de cargas do solo, avaliadas por meio de filmagem. Os autores descreveram brevemente o procedimento de validação do instrumento, sendo verificada alta reprodutibilidade interavaliador. Porém os autores não apresentam o teste estatístico tampouco o tamanho da amostra utilizada para validar o instrumento. Outro ponto crítico da aplicação do instrumento é de que, apesar dos escolares terem sido avaliados individualmente, esta avaliação foi na presença dos outros escolares, resultando em um viés de avaliação, pois a postura de um escolar pode ter influenciado os demais.

Já na década 90, Robertson e Lee (30), em seu estudo com 91 escolares de 10 a 12 anos de idade, desenvolveram uma escola postural com três aulas. Para as avaliações no pré e pós-teste, foram avaliadas a postura de sentar e de pegar objeto do solo, a partir de filmagem. Para cada tarefa realizada foram estipulados critérios necessários para uma postura adequada sentada e ao pegar objeto do solo. Porém, os autores não apresentam em nenhum momento o procedimento de validação deste protocolo de avaliação.

Em ambos os estudos $(14,30)$ o procedimento de análise das posturas "pegar objeto do solo e sentar" é de fácil entendimento e quantitativo, uma vez que a soma dos vários itens fornece a pontuação total de cada postura, a qual, por sua vez, representa a postura adequada. Entretanto, estes estudos analisam apenas a postura de pegar objeto do solo e sentar, sendo que os escolares realizam outras posturas, por igual ou até maior tempo, como sentar e utilizar o computador, dormir e transportar o material escolar.

Ainda na década de 90, no Brasil, Rocha e Souza (41) realizaram um estudo com o objetivo de apresentar a metodologia, sua validade e os coeficientes de reprodutibilidade de um instrumento de avaliação da postura dinâmica. Os participantes 
analisados cumpriram uma rotina de movimentos, sendo filmados por um observador em uma sala previamente preparada para sua realização. A rotina foi explicada antes de iniciar a filmagem, e durante a sua realização o observador forneceu aos participantes apenas as instruções necessárias sobre a sequência de movimentos. 0 instrumento, com o objetivo de avaliar de zero a quatro pontos a postura durante a execução de seis AVDs, foi submetido à validação de conteúdo por três especialistas em postura corporal. Os autores também realizaram, para verificar a consistência interna ${ }^{2}$ do instrumento e a reprodutibilidade intra e interavaliador, comparações de duas avaliações realizadas em dias diferentes por dois avaliadores, encontrando elevados índices de Alpha de Cronbach, de coeficiente intraclasse e de correlação de Spearman, respectivamente (Quadro 1). Esses resultados indicam que o instrumento proposto para análise da postura nas AVDs por meio de filmagem é coerente e fidedigno, podendo ser utilizado em estudos que objetivam avaliar a postura corporal dinâmica.

O instrumento proposto por Rocha e Souza (41), utilizado em estudos posteriores (Quadro 1), embora muito acessível e de fácil aplicação em qualquer contexto, foi validado com uma pequena amostra $(n=10)$, não sendo mencionado se fora realizado um cálculo amostral para escolha deste grupo de avaliados. Essa amostra foi composta por trabalhadores adultos, portanto, não é ideal que seja aplicado no ambiente escolar, pois não foi proposto com tal objetivo.

No ano de 2001, Vanderthommen et al. (45) publicaram um artigo com o objetivo de validar seu instrumento de avaliação da postura dinâmica proposto anteriormente (11). Assim, este estudo objetivou completar a primeira versão do teste ${ }^{3}$, adicionando novos critérios de avaliação, e validar essa nova versão. Para isso, foram avaliados três grupos: (a) pacientes que participaram de uma escola postural; (b) grupo controle; e (c) profissionais da área da saúde.
Como procedimentos de validação foram verificadas a sensibilidade do teste, a reprodutibilidade intra-avaliador, a reprodutibilidade interavaliador e a comparação dos escores provenientes de avaliações de três avaliadores para uma mesma avaliação. Os resultados demonstraram que esse instrumento de avaliação da postura dinâmica foi capaz de distinguir diferentes níveis de conhecimento, sendo sensível ao avaliar grupos com desempenhos distintos, pois os profissionais da saúde apresentaram escores superiores em comparação com o grupo controle e de pacientes. Da mesma forma, os coeficientes de correlação intraclasse, tanto para reprodutibilidade intra quanto interavaliador, foram elevados (Quadro 1). A partir desses resultados, os autores concluíram que esse instrumento representa uma ferramenta interessante para a avaliação da postura em hospitais e escolas posturais. Entretanto, dentre os procedimentos de validação, não está contemplada a avaliação da reprodutibilidade, ou seja, de teste e re-teste, e não é mencionada a realização de um cálculo amostral para escolha do tamanho amostral.

Cardon et al. (15) também desenvolveram, no mesmo período, um estudo similar. Percebe-se, desse modo, um investimento dos pesquisadores em fornecer meios confiáveis de avaliação da postura nas AVDs. A metodologia proposta por Cardon et al. (15), utilizada nos protocolos de avaliação em suas publicações posteriores (Quadro 1), avalia a postura dinâmica de seis AVDs: tirar os calçados, sentar ao escrever, pegar um objeto pesado do solo, pegar um objeto leve do solo, transportar o objeto, e transportar mochila escolar. Esse teste prático foi desenvolvido baseado em estudos anteriores com metodologias similares $(14,30)$ já discutidos anteriormente. Participaram 50 escolares de ambos os gêneros, com média de idade de dez anos, os quais percorreram o teste individualmente, sendo simultaneamente filmados. Cada postura foi analisada a

\footnotetext{
2 Representa a proporção da variabilidade nas respostas dos avaliadores, ou seja, permite verificar se as variabilidades encontradas são consequências das mudanças naturais de uma postura para outra. É comumente avaliada utilizando o coeficiente Alpha de Cronbach (51).

3 O mesmo grupo de pesquisadores, em estudo anterior (11), propôs um instrumento para avaliar a postura de pacientes com dor lombar. Nesse instrumento são avaliadas três posturas - deitar na cama, varrer e pegar objeto do solo - a partir de critérios pré-definidos, os quais variam de zero a oito pontos. Esse instrumento serviu de base para as recomendações da Société Belge des Écoles du Dos (SBED) (52), a qual propôs o seu uso e de outras duas tarefas adicionais - amarrar calçado e organizar uma estante. Entretanto, em ambas as publicações, embora fosse proposto o uso dessa ferramenta para avaliar a postura de pacientes com dor lombar, não foram apresentados dados referentes à validação do instrumento. Da mesma forma, ambas as publicações (11, 52) referiram a necessidade da validação do instrumento.
} 
partir de critérios pré-definidos, que variavam de 0 a 32 pontos. Entretanto, esta nota apresenta maior subjetividade na análise de cada um dos critérios de pontuação, visto que segue o seguinte princípio: quanto mais adequada a postura, mais a nota se aproxima do máximo da pontuação para aquela postura, não havendo critérios específicos para realização da pontuação, o que dificulta a aplicação dessa metodologia em outros estudos.

Os resultados do procedimento de reprodutibilidade interavaliador desse estudo (15) demonstraram correlações elevadas e significativas (Quadro 1). Entretanto, mesmo demonstrando alta correlação intraclasse, a determinação da pontuação é dependente principalmente da experiência e subjetividade do pesquisador. Além disso, assim como nos estudos anteriores, esse instrumento de avaliação da postura não contempla posturas realizadas com muita frequência e por longos períodos do dia pelos escolares, como a postura de dormir e utilizar o computador.

Também no ano de 2001, Méndez e Gómez-Conesa (13) realizaram um estudo com um grupo de escolares de ambos os sexos com o objetivo de verificar o efeito de um programa de educação postural que pretendia aumentar o nível de conhecimento sobre aspectos relacionados à postura corporal. Para a avaliação dos efeitos desse programa de ensino, um dos instrumentos utilizados foi o de avaliação da postura dinâmica, em que foram avaliadas as posturas, durante a execução de uma série de movimentos, por dois estudantes de fisioterapia. Os procedimentos de validação do instrumento, embora descritos brevemente, demonstraram alta reprodutibilidade interavaliador, ou seja, escores similares entre as avaliações de dois avaliadores. Entretanto, esse instrumento (13), assim como o instrumento proposto por Cardon et al. (15), não apresenta critérios quantitativos claros, que sejam independentes da subjetividade do avaliador, ou seja, não esclarece o método de pontuação de cada movimento.

O instrumento de avaliação proposto por Méndez e Gómez-Conesa (13) baseia-se em uma pontuação que varia de zero a dois pontos, sendo que o escolar que executa a ação sem causar estresse para a coluna vertebral receberia dois pontos; se o desempenho fosse só em parte satisfatório receberia um ponto; e se o movimento fosse totalmente insatisfatório não marcaria nenhum ponto. Entretanto, questiona-se esse método de pontuação, porque está sujeito a equívocos e diferentes interpretações de um gesto motor, já que depende da experiência do avaliador. Essas metodologias, dependentes da subjetividade e experiência do avaliador, são difíceis de serem reproduzidas em estudos posteriores. Ainda, nesse tipo de pontuação, não fica claro quando um movimento é satisfatório e não gera estresse à coluna vertebral, ou quando causa altos índices de estresse à coluna vertebral.

Em 2004, Karahan e Bayraktar (2) realizaram um estudo com o objetivo de analisar a postura corporal em ambientes clínicos e a ocorrência de dor nas costas de enfermeiros. Os 56 trabalhadores foram observados no próprio ambiente de trabalho, sendo sua postura analisada a partir de critérios biomecânicos pré-definidos. Foram nove as AVDs contempladas: postura sentada e em pé, postura ao pegar objeto do solo, transportá-lo e trocá-lo de lugar, postura ao empurrar objetos e posturas ao mover um paciente na cama, mover paciente para posição sentada e em pé. Entretanto, tal método de avaliação proposto não apresenta nenhuma informação de procedimentos de validação, sendo apenas utilizado no próprio estudo.

A proposta mais recente de avaliação da postura durante as AVDs encontrada na literatura foi desenvolvida por Furtado et al. (50). Tal estudo objetivou traduzir e adaptar o instrumento proposto por Vanderthommen et al. (45), bem como avaliar a sua reprodutibilidade e validade de construto para utilização com a população brasileira. Como já descrito anteriormente, compõem esse instrumento cinco AVDs: deitar na cama, varrer, pegar objeto do solo, amarrar calçados e organizar uma estante. Participaram desse estudo de validação 30 pacientes com média de idade de 50 anos, com presença de dor lombar e 15 profissionais da área da saúde. Os resultados da reprodutibilidade inter e intra-avaliador foram elevados para todas as AVDs, apresentando valores significativos de correlação (Quadro 1). Do mesmo modo, os resultados referentes à validade de constructo (comparação dos escores do grupo de profissionais com o de pacientes) demonstraram que o instrumento é sensível para distinguir duas populações com diferentes níveis de conhecimento de execução adequada de AVDs. Desse modo, esse instrumento demonstra ser aplicável, de fácil manuseio, baixo custo, reprodutível e válido para a avaliação de pacientes com dor lombar. 


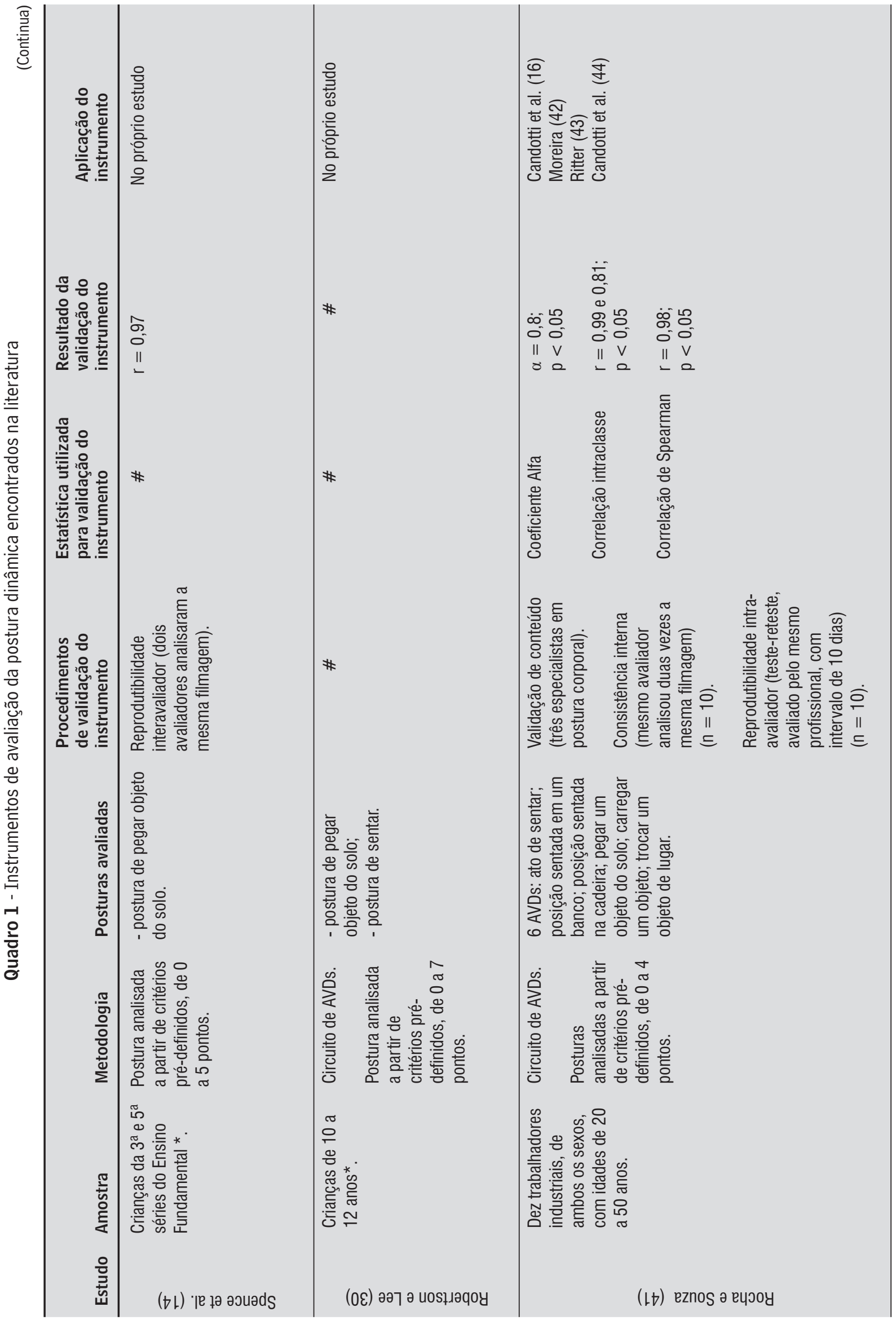




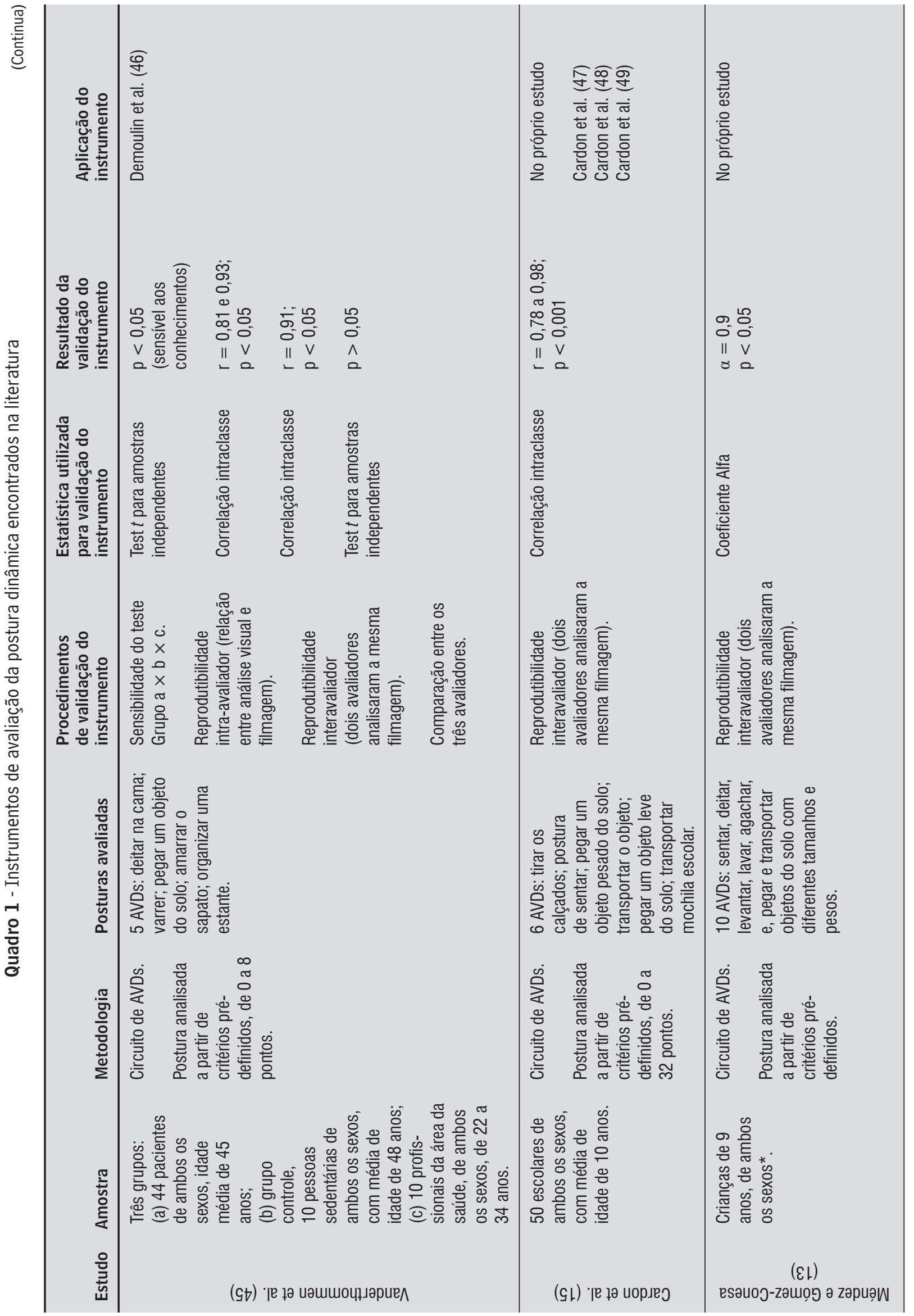




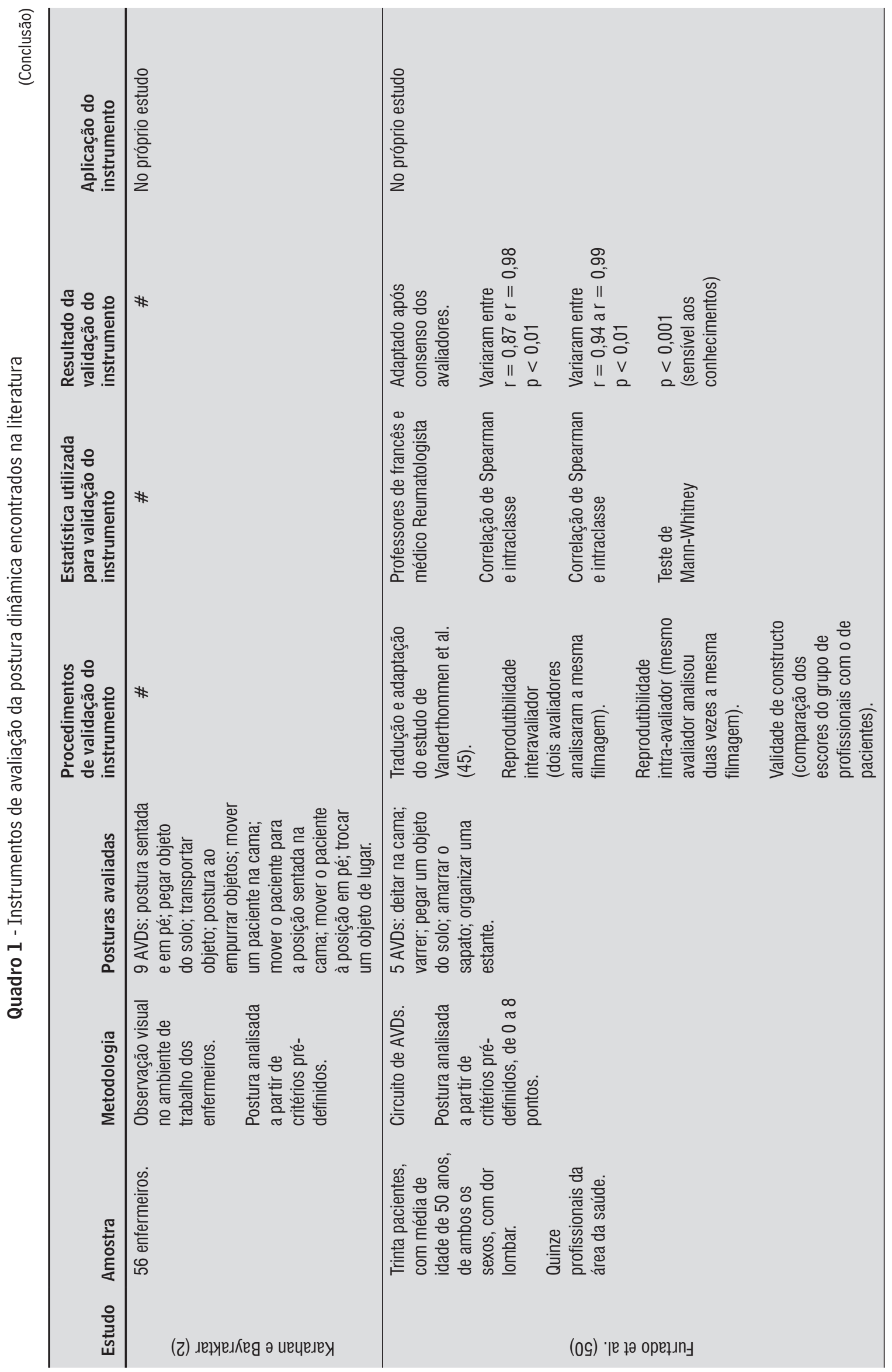


Posturas analisadas e critérios de avaliação

Os oito instrumentos de avaliação da postura dinâmica abordados, dentre a totalidade das posturas analisadas, objetivam avaliar algumas posturas em comum, tais como: postura sentada, postura ao pegar e ao transportar objetos. Um dos motivos pelos quais essas posturas são focadas por esses pesquisadores que desenvolveram os instrumentos de avaliação, possivelmente, é a possibilidade de tais posturas estarem associadas à ocorrência de dor e de sobrecargas da estrutura muscular e articular. Straker (53), por exemplo, afirma que uma técnica inadequada de agachamento ao erguer objetos do solo gera um aumento das forças de cisalhamento na coluna vertebral e do estresse ligamentar em torno de $50 \%$ e $75 \%$ quando comparada à técnica adequada de agachamento, ou seja, com flexão de joelhos e quadril e mínima flexão do tronco. Do mesmo modo, conforme Wilke et al. (37), segurar o objeto próximo ao corpo pode reduzir a pressão intradiscal em torno de $50 \%$, em comparação com o objeto distante do corpo. Já em posturas com o tronco inclinado à frente e com o objeto distante do centro de massa do indivíduo, a pressão intradiscal é aumentada em aproximadamente 4,5 vezes em relação à posição ortostática (37).

A postura sentada também recebeu até então uma maior preocupação dos pesquisadores; possivelmente, porque a grande maioria dos indivíduos, tanto na infância e adolescência como na fase adulta, permanece nessa postura por aproximadamente oito horas por dia (54). Além disso, estudos têm demonstrado que a permanência nessa postura por um longo período sem pausa predispõe dor e fadiga, em diferentes partes do corpo e, principalmente, a processos degenerativos, como a hérnia de disco (37, 55). Essa situação associada à postura inadequada (flexão anterior do tronco, falta de apoio lombar e falta de apoio do antebraço) tende a acentuar esses malefícios (54).

Tais instrumentos que objetivam avaliar a postura dinâmica durante a execução de AVDs utilizam critérios pré-definidos por escalas numéricas desenvolvidas a partir de conceitos biomecânicos, como exemplificado no Quadro 2. Esse quadro ilustra os critérios biomecânicos em comum entre cinco dos instrumentos anteriormente descritos $(14,30,41,45$, 50). Assim, a partir de critérios objetivos e definição clara do que é considerada uma postura adequada e inadequada, é possível diminuir a margem de subjetividade e possibilitar que a análise proposta pelo instrumento tenha melhores índices de reprodutibilidade intra e interavaliador, sendo capaz de fornecer resultados reprodutíveis, seja no âmbito da pesquisa ou do acompanhamento da postura de escolares.

No entanto, de modo geral, algumas limitações são identificadas nos instrumentos apresentados. A primeira e mais contundente refere-se à necessidade de avaliar outras posturas, como a postura de dormir, sentar para exercer outras AVDs, e outras atividades domésticas, tanto para a população de adultos como para escolares.

Outra limitação diz respeito ao fato de que os instrumentos descritos, que propõem a avaliação da postura dinâmica, não definem o momento exato para que o avaliador realize a pontuação para cada postura. Por exemplo, o ato de sentar, engloba o início do movimento até o final do movimento sobre o assento, e os instrumentos não especificam em qual momento do movimento é realizada a análise. Isso ocorre também em outros movimentos, como por exemplo, no ato de pegar objeto do solo, onde não é especificado se a análise é realizada durante o deslocamento do corpo em direção ao objeto, no momento de contato do corpo e objeto, ou no momento de retirada do objeto do solo. Essa indefinição aumenta a possibilidade de erro intra e interavaliador e dá margem para que um mesmo instrumento seja utilizado de diferentes formas.

Quadro 2 - Posturas e os respectivos critérios biomecânicos para avaliação, comuns a maioria dos estudos

\begin{tabular}{ll}
\hline Postura & $\begin{array}{l}\text { Critério biomecânico para análise } \\
\text { da postura }\end{array}$ \\
\hline Postura sentada & $\begin{array}{l}\text { Manter as curvaturas fisiológicas da } \\
\text { coluna vertebral. } \\
\text { Membros inferiores afastados e simétricos. } \\
\end{array}$ \\
& $\begin{array}{l}\text { Flexão de joelhos e quadril de } 90 \text { graus. } \\
\text { Sola dos pés apoiados em uma base ou } \\
\text { no solo. }\end{array}$ \\
\hline Postura ao pegar & Manter as curvaturas fisiológicas da \\
um objeto do & coluna vertebral. \\
solo & Membros inferiores afastados e simétricos. \\
& Flexão de joelhos e quadril. \\
& Objeto próximo ao corpo. \\
\hline Postura ao & Manter as curvaturas fisiológicas da \\
transportar & coluna vertebral. \\
objetos & Não elevar os ombros. \\
& Objeto próximo ao corpo. \\
\hline
\end{tabular}

Fonte: Dados da pesquisa. 
Avaliação Dinâmica em escolares

A necessidade de realizar avaliações da postura dinâmica faz que profissionais da área da saúde, assim como os pesquisadores, optem, dentre esses oito instrumentos disponíveis na literatura, pelo mais aplicável em seu contexto. No entanto, referente ao contexto escolar, esses instrumentos que objetivam avaliar a postura dinâmica durante a execução de AVDs não contemplam outras AVDs realizadas com frequência pelos escolares, como a postura sentada no computador e a postura ao transportar material escolar.

Como demonstrado por Robbins et al. (55), a ocorrência de dor nas costas está associada à utilização de computadores, provavelmente devido à postura inadequada. Isso justifica a necessidade de avaliar, além das posturas comumente avaliadas, a postura corporal no manuseio do computador, e estar atento também ao tempo de utilização do equipamento. Os dados publicados pelo Instituto Brasileiro de Geografia e Estatística (IBGE) referentes ao ano de 2009 demonstraram que $41 \%$ dos domicílios do estado Rio Grande do Sul, e 34,7\% dos domicílios do Brasil já possuem computador (56). Os dados longitudinais demonstram que a presença do computador nas moradias dos cidadãos brasileiros cresce a cada ano (56). Do mesmo modo, especula-se que os jovens que não possuem computadores em suas moradias provavelmente os utilizam nas escolas ou em centros de lazer.

Na última década, além do uso de computadores de mesa, tem sido usual também o uso de computadores portáteis (laptop) pelos escolares. Straker et al. (57) referem que o uso de computadores portáteis, por suas características, induz a uma postura com flexão cervical aumentada, resultando em maior tensão da região cervical e dos ombros.

Da mesma forma que a dor e as alterações posturais podem estar associadas ao uso de computadores, estudos demonstram que o uso inadequado de mochilas escolares também pode estar associado a esses malefícios (19). Entretanto, escolares, pais e educadores acabam esquecendo o perigo do transporte de materiais de modo inadequado e com excesso de peso (19). Conforme Skoffer (58), a sobrecarga resultante do trasnporte da mochila pode contribuir para um aumento da prevalência de sintomas musculoesqueléticos em escolares. Do mesmo modo, a maneira de carregar a mochila pode ser um fator de risco para a ocorrência de dor nas costas e ombros $(59,60)$. Segundo Korovessis et al. (61), isso pode ocorrer por causa de um aumento das tensões aplicadas nas estruturas da coluna vertebral e por alterações na postura e na marcha dos escolares (62). Nessa perspectiva, os instrumentos de avaliação da postura corporal dinâmica devem ser ampliados para que possam avaliar um maior número de AVDs específicas do contexto de vida dos escolares.

Não obstante, a aplicabilidade dos instrumentos de avaliação da execução de AVDs baseados na observação e filmagem no contexto escolar é potencialmente dependente de vários aspectos, tais como: físicos, temporais, recursos financeiros, recursos humanos e projetos pedagógicos. Os aspectos físicos relacionam-se às questões do espaço e dos materiais necessários para a montagem das estações dos circuitos de AVDs. Os aspectos temporais referem-se à demanda de tempo que esse tipo de avaliação requer, seja para a filmagem em si ou para a análise dessa filmagem. Os recursos financeiros envolvem a necessidade de aquisição de equipamentos como filmadora e tripé; já os recursos humanos relacionam-se à necessidade de a escola ter profissionais capacitados tanto para a proposição e execução das avaliações quanto para realizar a análise e interpretação dos resultados, transformando-os em informação útil que subsidie a prática da disciplina de Educação Física (63).

Por fim, os projetos pedagógicos das escolas devem suportar ações dessa natureza, permitindo a aplicação dessas avaliações no contexto formal da disciplina de Educação Física ou incentivando o desenvolvimento de projetos ou programas de educação postural (PEP). Considerando que a literatura já evidencia os efeitos benéficos dos PEP (14-16, 19, 20, 47-49) sobre a postura dos escolares, entende-se que a descrição, a sintetização e a análise crítica dos instrumentos de avaliação da postura dinâmica realizadas na presente revisão podem contribuir para a adequação, o acompanhamento e o planejamento anual de PEP em escolas; estes podem ser desenvolvidos no contexto formal da disciplina de Educação Física ou em turnos inversos.

\section{Considerações finais}

A publicação oficial das escolas de coluna da Bélgica, Recommandations de la Société Belge des Écoles du Dos (SBED) (52), descreve que os métodos mais utilizados na avaliação da postura são: questionários de sintomas musculoesqueléticos (68\%); 
escala visual análoga de dor (EVA) (43\%); equilíbrio muscular dos músculos do tronco (37\%); teste prático de avaliação da execução de AVDs a partir de filmagem (31\%); e teste prático de avaliação da execução de AVDs a partir de observação visual (12\%) (52). Esses dados demonstram que o uso do questionário se sobressai aos demais métodos, provavelmente pela facilidade de obtenção das informações. Entretanto, os questionários em geral não são capazes de mensurar a incorporação do conhecimento teórico na prática, ou seja, a transferência do conhecimento para a execução do movimento, fato facilmente observado na filmagem da postura dinâmica (14). Possivelmente, por esse motivo vários pesquisadores $(14,31,45)$, na busca por avaliações de qualidade, têm apontado a necessidade do uso concomitante de testes dinâmicos padronizados para avaliação da postura dinâmica.

Outro fator que estimula a utilização dos testes dinâmicos de avaliação da postura relaciona-se com a possibilidade de serem facilmente realizados em diferentes contextos, já que é possível avaliar qualquer indivíduo fora do ambiente de laboratório (41). Entretanto, para que a investigação da postura dinâmica seja efetivada, são necessárias metodologias validadas e apropriadas para esse fim. Nesse sentido, os protocolos de avaliação da postura dinâmica citados no presente artigo devem ser utilizados com ressalvas em avaliações de escolares, pois de forma geral, apresentam alguns problemas metodológicos: não descrevem os procedimentos que demonstram sua reprodutibilidade; não demonstram os procedimentos estatísticos bem delineados e não apresentam os cálculos para a definição do tamanho da amostra.

Ainda, dentre os instrumentos descritos, apenas quatro foram elaborados com o propósito de avaliar algumas das AVDs de escolares, o que evidencia a necessidade do desenvolvimento de instrumentos específicos para essa população que apresentem amostra relevante, validade e índices satisfatórios de repetibilidade, reprodutibilidade inter e intra-avaliador com o objetivo de elevar a qualidade da avaliação da postura dinâmica de escolares.

\section{Referências}

1. Candotti CT, Noll M, Cruz M. Prevalência de dor lombar e os desequilíbrios musculares em manicures. Rev. Arquivos Mov. 2010;6(1):125-40.
2. Karahan A, Bayraktar N. Determination of the usage of body mechanics in clinical settings and the occurrence of low back pain in nurses. Int J Nurs Stud. 2004;41:67-75. doi:10.1016/S0020-7489(03)00083-X.

3. Limon S, Valinsky LJ, Shalom YB. Risk factors for low back pain in the elementary school environment. Spine. 2004;29(6):697-702. doi:10.1097/01. BRS.0000116695.09697.22.

4. Ribeiro CC, Gómez Conesa A. Lower back pain: prevalence and preventive programs in childhood and adolescence. Rev Iberoam Fisioter Kinesiol. 2008; 11(1):32-8.

5. Correa A, Pereira JS, Silva MA. Avaliação dos desvios posturais em escolares: estudo preliminar. Rev Fisioter Bras. 2005;6(3):175-8.

6. Paananen MV, Taimela SP, Auvinen JP, Tammelin TH, Kantomaa MT, Ebeling H, et al. Risk factors for persistence of multiple musculoskeletal pains in adolescence: A 2-year follow-up study. Eur J Pain. 2010;14:1026-32. doi:10.1016/j.ejpain.2010.03.011.

7. Szpalski M, Gunzburg R, Balagué R, Nordin M, Mélot C. A 2year prospective longitudinal study on low pain in primary school children. Eur Spine J. 2002;11:459-64. doi:10.1007/s00586-002-0385-y.

8. Detsch C, Luz AM, Candotti O, Lazaron F, Guimaraes L, Schimanoski P. Prevalência de alterações posturais em escolares do ensino médio em uma cidade no Sul do Brasil. Rev Panam Salud Publica. 2007;21(4):231-8. doi:10.1590/S1020-49892007000300006.

9. Siivola S, Levoska S, Latvala K, Hoskio, E, Vanharanta H, Kiukaanniemi SK. Predictive Factors for Neck and Shoulder Pain: A longitudinal Study in Young Adults. Spine. 2004;29(15):1662-9. doi:10.1097/01. BRS.0000133644.29390.43.

10. Trevelyan FC, Legg SJ. Back pain in school children Where to from here? App Ergonomics. 2006;37:45-54. doi:10.1016/j.apergo.2004.02.008.

11. Vanderthommen M, Defaweux M, Tomasella M, Crielaard JM. Le comportement gestual du patient lobalgique fréquentant une école du dos: analyse préliminaire dún test d'évaluation. Annales de Réadaption et de Médecine Physique. 1999;42:485-92. doi:10.1016/ S0168-6054(00)87702-8. 
12. Paananen MV, Auvinen JP, Taimela SP, Tammelin TH, Kantomaa MT, Ebeling HE, et al. Phychosocial, mechanical, and metabolic factors in adolescents' musculoskeletal pain in multiple locations: a Cross-sectional study. Eur J Pain. 2010;14:395-401. doi:10.1016/j. ejpain.2009.06.003.

13. Méndez FJ, Gómez-Conesa A. Postural hygiene program to prevent low back pain. Spine. 2001;26(11):1280-6. doi:10.1097/00007632-200106010-00022.

14. Spence SM, Jensen GM, Shepard KF. Comparison of methods of teaching children proper lifting techniques. Phys Ther. 1984;64(7):1055-66. PMid:6739547.

15. Cardon G, Clercq D, Bourdeaudhuij I. Effects of back care education in elementary schoolchildren. Acta Paediatric. 2000;(89):1010-7. doi:10.1111/j.16512227.2000.tb00426.x.

16. Candotti CT, Macedo CH, Noll M, Freitas K. Escola postural: uma metodologia adaptada para crianças. Rev Arquivos Mov. 2009;5(2):34-49.

17. Auvinen JP, Tammelin TH, Taimela SP, Zitting PJ, Jarvelin M, Taanila AM, et al. Is insufficient quantity and quality of sleep a risk factor for neck, shoulder and low back pain? A longitudinal study among adolescents. Spine. 2010;19:641-9. doi:10.1007/ s00586-009-1215-2.

18. Stevenson JM, Weber CL, Smith T. A longitudinal study of the development of low back pain in an industrial population. Spine. 2001;26(12):1370-7. doi:10.1097/00007632-200106150-00022.

19. Candotti CT, Roth E, Noll M. Evaluation of weight and mode of transport of student in school of education. Rev Paul Pediatr. 2012;30(1):100-106. doi:10.1590/ S0103-05822012000100015.

20. Candotti CT, Nunes SE, Noll M, Freitas K, Macedo CH. Efeitos de um programa de educação postural para crianças e adolescentes após oito meses do seu término. Rev Paul Pediatr. 2011;29(4):577-583. doi:10.1590/S0103-05822011000400017.

21. Neuschwander T, Cutrone J, Macias B, Cutrone S, Chambers H, Hargens A. The effect of backpacks on the lumbar spine in children: a standing magnetic resonance imaging study. Spine. 2009;35(1):83-8. doi:10.1097/BRS.0b013e3181b21a5d.
22. Kellis E, Emmanouilidou M. The effects of age and gender on the weight and use of schoolbags. Pediatr Phys Ther. 2010;22:17-45. doi:10.1097/PEP. 0b013e3181cbf852.

23. Haan RJ. Measuring quality of life after stroke using the SF-36. Stroke. 2002;33:1176-1177. doi:10.1161/01. STR.0000015223.98452.97.

24. Borges R, Vieira A, Noll M, Bartz P, Candotti CT. Efeitos da participação em um Grupo de Coluna sobre as dores musculoesqueléticas, qualidade de vida e funcionalidade dos usuários de uma Unidade Básica de Saúde de Porto Alegre - Brasil. Motriz. 2011;17(4):719-27. doi:10.1590/S1980-65742011000400017.

25. Melzack R. The McGill Pain Questionnaire. Anesthesiology. 2005;103(1):199-202. doi:10.1097/ 00000542-200507000-00028.

26. Vigatto R, Alexandre NMC, Correa HR Filho. Development of a brazilian portuguese version of the oswestry disability index: cross-cultural adaptation, reliability, and validity. Spine. 2007;32(4):481-6. doi:10.1097/01.brs.0000255075.11496.47.

27. Nusbaum L, Natour J, Ferraz MB, Goldenberg J. Translation, adaptation and validation of the Roland-Morris questionnaire - Brazil Roland-Morris. Braz J Med Biol Res. 2001;34:203-10. doi:10.1590/ S0100-879X2001000200007.

28. Vidal J, Borras PA, Ortega FB, Cantallops J, Ponseti S, Palou P. Effects of postural education on daily habits in children. Int J Sports Med. 2011;32:303-8. doi:10.1055/s-0030-1270469.

29. Park JH, Kim JS. Effects of spinal health educational programs for elementary school children. J Spec Pediatr Nurs. 2011;16:121-9. doi:10.1111/ j.17446155.2011.00278.x.

30. Robertson HC, Lee VC. Effects of back care lessons on sitting and lifting by primary students. Aust Physiother. 1990;36(4):245-8.

31. Andreotti RA, Okuma SS. Validação de uma bateria de testes de atividades da vida diária para idosos fisicamente independentes. Rev Paul Educ Fís. 1999;13(1):46-66.

32. Thomas JR, Nelson JK, Silverman S. Métodos de pesquisa em atividade física. Porto Alegre: Artmed; 2012. 
33. Smith A, O'Sullivan P, Straker L. Classification of sagittal thoraco-lombro-pelvic alignment of the adolescent spine in standing and its relationship to low back pain. Spine. 2008;33(19):2101-17. doi:10.1097/ BRS.0b013e31817ec3b0.

34. O'Sullivan P, Grahamslaw KM, Kendell M, Lapenskie S, Moller NR, Richars K. The effect of different standing and sitting postures on trunk muscle activity in a pain-free population. Spine. 2002;27(11):1238-44. doi:10.1097/00007632-200206010-00019.

35. Rohlmann A, Graichen F, Kayser R, Bender A, Nat R, Bergmann $G$. Loads on a telemeterized vertebral body repkacement measured in two patients. Spine. 2008;33(11):1170-9. doi:10.1097/BRS. 0b013e3181722d52.

36. Nachemson A, Moris J. In vivo measurements of intradiscal pressure discometry, a method for the determination of pressure in the lower lumbar discs. J Bone Joint Surg Am. 1964;46:1077-92.

37. Wilke J, Neef P, Caimi M, Hoogland T, Claes L. New in vivo measurements of pressures in the intervertebral disc in daily life. Spine. 1999;24(8):755-62. doi:10.1097/00007632-199904150-00005.

38. Noll M, Candotti CT, Vieira A. Escola de Educação Postural: revisão sistemática dos programas desenvolvidos para escolares no Brasil. Movimento. 2012;18(4):265-91.

39. Associação Brasileira de Normas Técnicas. Norma Brasileira Registrada: NBR 10536 - Statistics: Vocabulary and Symbols; 1988.

40. Joint Committee for Guides in Metrology - JCGM. Working Group 2. International vocabulary of metrology: basic and general concepts and associated terms. 2008.

41. Rocha A, Souza JL. Observação das atividades de vida diária através de vídeo. Movimento. 1999;5(11):16-22.

42. Moreira S. Características da postura corporal de escolares da rede municipal de ensino de Porto Alegre [dissertação]. Porto Alegre: Mestrado em Ciências do Movimento Humano, Universidade Federal do Rio Grande do Sul; 2008.

43. Ritter AL. Postura corporal ao sentar e transportar material escolar [tese]. Porto Alegre: Doutorado em Ciências do Movimento Humano, Universidade Federal do Rio Grande do Sul; 2009.
44. Candotti CT, Macedo CH, Noll M, Freitas K. Escola de postura: uma metodologia adaptada aos pubescentes. Rev Mackenzie Educ Fís Esporte. 2010;9(2):91-100.

45. Vanderthommen M, Boulange AS, Defaweux M, Tomasella M, Crielaard JM. Validation d'un test d'évaluation du comportement gestuel du patient lombalgique chronique. Ann Réadapt Méd Phys. 2001;44(5):281-90. doi:10.1016/S0168-6054(01)00101-5.

46. Demoulin C, Grosdent S, Capron L, Tomasella P, Crielaard J, Vanderthommen M. Intérêt dúne prise em charge multidisciplinaire ambulatoire semi-intensive dans La lombalgie chronique. Rev Rhum. 2010;77:6873. doi:10.1016/j.rhum.2009.05.011.

47. Cardon G, Clercq D, Bourdeaudhuij I. Back care education in elementary school: a pilot study investigating the complementary role of the class teacher. Patient Educ Couns. 2001;45:219-26. doi:10.1016/ S0738-3991(01)00122-7.

48. Cardon G, Clercq D, Bourdeaudhuij I. Generalization of back education principles by elementary school children: evaluation with test and a candid camera observation. Acta Paediatric. 2001;90:143-50. doi:10.1111/j.1651-2227.2001.tb00275.x.

49. Cardon G, Clercq D, Bourdeaudhuij I. Back education efficacy in elementary schoolchildren: a 1-year follow-up study. Spine. 2002;27(3):299-305. doi:10.1097/ 00007632-200202010-00020.

50. Furtado R, Jones A, Furtado RV, Jennings F, Natour J. Validation of the brazilian-portuguese version of the gesture behavior test for patients with non-specific chronic low back pain. Clinics. 2009;64(2):83-90. doi:10.1590/S1807-59322009000200004.

51. Pestana MH, Gageiro JN. Análise de dados para ciências sociais. Edições Sílabo: Lisboa, 2003. PMCid:152652.

52. Henrotin Y, Vanderthommen M, Fauconnier C, Grisar J, Masquelier E, Peretz A, et al. Définition, critéres de qualité et evaluation d'un programme de type école du dos. Recommandations de la Société belge des écoles du dos. Rev Rhum. 2001;68(2):185-91. doi:10.1016/ S1169-8330(00)00095-8.

53. Straker L. Evidence to support using squat, semisquat and stoop techniques to lift low-lying objects. Int J Ind Ergon. 2003;31:149-60. doi:10.1016/ S0169-8141(02)00191-9. 
54. Womersley L, May S. Sitting posture of subjects with postural backache. J Manip Physiol Ther. 2006;29:213-8. doi:10.1016/j.jmpt.2006.01.002.

55. Robbins M, Johnson IP, Cunliffe C. Encouraging good posture in school children using computers. Clin Chiropractic. 2009;12:35-44. doi:10.1016/j.clch. 2009.03.003.

56. Instituto Brasileiro de Geografia e Estatística - IBGE. Pesquisa nacional por amostra de domicílios: síntese de indicadores 2009. Rio de Janeiro, 2010.

57. Straker L, Jones K, Miller J. A comparison of the postures assumed when using laptop computers and desktop computers. Appl Ergon. 1997;28(4):263-8. doi:10.1016/S0003-6870(96)00073-7.

58. Skoffer B. Low Back Pain in 15 to 16 year old children in relation to school furniture and carrying of the school bag. Spine. 2007;32(24):713-7. doi:10.1097/ BRS.0b013e31815a5a44.

59. Gent C, Dols JJ, Rover CM, Sing RA, Vet HC. The weight of schoolbags and the occurrence of neck, shoulder, and back pain in young adolescents. spine. 2003;28(9):916-21. doi:10.1097/01.BRS. 0000058721.69053.EC.
60. Mackie HW, Legg SJ, Beadle J, Hedderly D. Comparison of four different backpacks intended for school use. Appl Ergon. 2003;34:257-64. doi:10.1016/ S0003-6870(03)00034-6.

61. Korovessis P, Koureas G, Zacharatos S, Papazisis Z. Backpacks, back pain, sagittal spinal curves and trunk alignment in adolescents. Spine. 2005;30(2):247-55. doi:10.1097/01.brs.0000150505.59690.1b.

62. Whittfield J, Legg SJ, Hedderly DI. Schoolbag weight and musculoskeletal symptoms in New Zealand secondary schools. Appl Ergon. 2005;36:193-8. doi: 10.1016/j.apergo.2004.10.004.

63. Candotti C, Rohr J, Noll M. A educação postural como conteúdo curricular da Educação Física no Ensino Fundamental II nas escolas da Cidade de Montenegro/ RS. Movimento. 2011;17(3):57-77.

Recebido: 20/05/2012

Received: 05/20/2012

Aprovado: 06/11/2012

Approved: 11/06/2012 